\section{Hilfeplanung für chronisch mehr- fachbeeinträchtigt Abhängige - zwischen Anspruch und Wirklichkeit}

Martina Schu, Günter Schlanstedt, Hans Oliva

FOGS - Gesellschaft für Forschung und Beratung im

Gesundheits- und Sozialbereich, Köln
Zusammenfassung: Im Rahmen des vom BMG geförderten Kooperationsmodells nachgehende Sozialarbeit wurde fünf Jahre lang Case Management für chronisch mehrfachbeeinträchtigt Abhängige erprobt. Im Mittelpunkt dieses Beitrags stehen die Erfahrungen mit der Umsetzung von Hilfeplanung und -abstimmung unter Verwendung eines formalisierten Verfahrens.

Die Erprobung zeigte, dass das Kernelement von Case Management, die Hilfeplanung, ein geeignetes System für umfassende Planung und Klientenbeteiligung sowie die fallbezogene Steuerung und Abstimmung von Hilfen darstellt. Gerade in besonders schwierigen und chaotischen Fällen konnte Hilfeplanung helfen, den Überblick zu behalten und die Verbindlichkeit von Absprachen zu steigern. Hilfeplanung erwies sich darüber hinaus als ein gutes Instrument zu Qualitätsentwicklung und -sicherung, indem Sinnhaftigkeit, Angemessenheit und Effektivität der eigenen Leistungen sowie der weiteren Planungen und Vermittlungen laufend überprüft werden.

Allerdings verlangen Hilfeplanung und -abstimmung, Monitoring, die Übernahme von Fallverantwortung sowie Ressourcenorientierung ein verändertes Selbstverständnis der Helfer, ein hohes Maß an Bereitschaft und Selbstreflexion. Weil jedoch traditionelles eher angebotszentriertes Professionsverständnis noch überwiegt und neue sozialarbeiterische Ansätze wenig geübt sind, stieß die Einführung von Hilfeplanung bei einem großen Teil der Modellprojekte zuerst und z.T. dauerhaft auf größere Schwierigkeiten (schriftliche Fixierung, Zieldefinition, Hilfeabstimmung, Hilfekonferenzen, Monitoring etc.).

Schlüsselwörter: Case Management - Hilfeplanung Hilfekoordination - Suchtkrankenhilfe - Drogenhilfe Sozialarbeit

Aid Planning for Chronically Multi-Impaired Addicts Between Vision and Reality: As part of the „Cooperation Pilot Project of Follow-up Social Work“, sponsored by Germany's Federal Ministry for Health (BMG), a case management scheme for „chronically multi-impaired addicts“ was trialed for five years. This paper focuses on the experience gained implementing aid support and coordination under a formalised procedure.

Suchttherapie 2001; 2: 65-72

(c) Georg Thieme Verlag Stuttgart · New York

ISSN 1439-9903
The pilot project demonstrated that the core element of case management, namely aid planning, is a suitable system for evolving more comprehensive planning and client participation, together with case-based aid management and coordination. Particularly when dealing with highly problematic and chaotic cases, aid planning can help to maintain an overview and enhances compliance with agreements. In addition, aid planning has proved itself to be an effective instrument for ensuring quality development and quality assurance through the ongoing appraisal of the raison d'être, suitability and efficacy of services, and further planning and referrals.

However, aid planning and coordination, monitoring, acceptance of case management and resource-orientation demands a change of self-perception among aid workers, allied to a high degree of motivation and self-reflection. Since, however, a more traditional, service-orientated professional image still predominates and new welfare approaches have been rarely attempted, the introduction of aid planning in a majority of pilot projects met both initially, and partly over the longer term, with great difficulties (written case notes, target definition, aid coordination, aid conferences, monitoring etc.).

Key words: Case-Management - Aid Planning - Aid Coordination - Addict Aid - Drugs Aid - Social Work

\section{Einleitung}

Im Rahmen des vom Bundesministerium für Gesundheit geförderten Kooperationsmodells nachgehende Sozialarbeit wurde von 1995 bis 2000 Case Management/nachgehende Sozialarbeit für die Zielgruppe der chronisch mehrfachbeeinträchtigt Abhängigen (CMA) erprobt. Die Arbeit der modellgeförderten Case Manager zielte neben der besseren Erreichung von CMA insbesondere auf die Optimierung der Hilfeerbringung durch Vermittlung, einzelfallbezogene Hilfeplanung und -koordination [1].

Im Kooperationsmodell arbeiteten 33 Case Manager in den alten und neuen Bundesländern, die Stellen waren überwiegend in Sucht- oder Drogenberatungsstellen und niedrigschwelligen Angeboten freigemeinnütziger Träger sowie seltener in sozialpsychiatrischen Diensten von Kommunen angebunden.

In der Fachdiskussion werden unter dem Begriff Case Management verschiedene Ansätze subsumiert, gleichwohl gelten einheitlich übergreifende Kernelemente: Case Manage- 
ment wird als Verknüpfungsaufgabe interpretiert, bei der Menschen mit individuellen Problemlagen und Hilfebedarfen (Nachfrageseite) und verfügbare Hilfemöglichkeiten und -instanzen (Angebotsseite) zusammengeführt werden [2]. Auch wenn die vielfältigen Konzepte keine einheitliche Auffassung zum Ausmaß der unmittelbaren Hilfeerbringung durch die Case Manager vertreten, besteht doch Einigkeit darüber, dass - im Unterschied zur klassischen Einzelfallhilfe - Case Manager die notwendigen Hilfeleistungen größtenteils nicht selbst erbringen, sondern sie vielmehr organisieren. Der Prozess des Case Managements ist stark strukturiert und umfasst die Arbeitsschritte Assessment (mit einer Bestandsaufnahme von Situation, Unterstützungsbedarfen und Ressourcen des Klienten), Hilfeplanung (mit der Festlegung von Zielen, Vorgehen und Zuständigkeiten), Durchführung (mit Erschließung, Organisation und Koordination aller notwendigen Hilfen), laufender Fallkontrolle (Monitoring) sowie der abschließenden Auswertung/Evaluation des Hilfeprozesses.

In der Umsetzung von Case Management kommt der Hilfeplanung ein herausgehobener Stellenwert zu, denn sie stellt die Grundlage für die gemeinsame Arbeit, die Steuerung des Gesamthilfegeschehens, die laufende Fallkontrolle und die (abschließende) Bewertung des Betreuungsverlaufs dar. Im Rahmen dieser Kernaufgabe von Case Management werden erforderliche und geeignete Angebote identifiziert sowie $\mathrm{Zu}$ ständigkeiten und Vereinbarungen über das konkrete Vorgehen festgelegt. Im Idealfall geschieht dies in einer Hilfeplankonferenz gemeinsam mit Case Manager und Klient sowie allen weiteren beteiligten, professionellen und privaten Helfern. In jedem Fall sollte der Hilfeplan das Ergebnis ausgehandelter Übereinkünfte zwischen Case Manager und Klient sein und eine Art „Gesamtplan“ darstellen, in dem nach Prioritäten geordnete Ziele, operationalisierbare Arbeitsschritte, Aufgabenverteilung und Zeitvorgaben festgelegt sind.

Im Rahmen der wissenschaftlichen Begleitung des Kooperationsmodells wurde die Logik des Case-Management-Prozesses in ein Dokumentationsinstrument übersetzt, dem mehrere Funktionen zukamen: Zum einen sollten die Teilschritte der Hilfeplanung so abgebildet werden, dass das Instrument die Modellmitarbeiter in der Durchführung unterstützen und gleichzeitig ihnen einen systematischen Rahmen für die Planung bieten sollte. Zum anderen waren die Belange der Evaluation sicherzustellen. Vor diesem Hintergrund entstand in enger Zusammenarbeit mit den Case Managern das integrierte Hilfeplanungs- und Dokumentationsinstrumentarium.

Anknüpfend an diese einleitenden Bemerkungen sollen nachfolgend erste Ergebnisse zur Umsetzung des Hilfeplanverfahrens - im Zeitraum von 1997 bis 1999 - im Kooperationsmodell nachgehende Sozialarbeit vorgestellt und diskutiert werden ${ }^{1}$.

\section{Einsatz der Hilfeplanung im Modellprogramm}

Wie bereits ausgeführt, stellt die Hilfeplanung das Kernelement von Case Management dar. Mit ihr rückt - verglichen mit traditioneller Sozialarbeit - eine andere/neue Hilfephi-

\footnotetext{
${ }^{1}$ Der vorliegende Beitrag muss sich auf die Darstellung von Zwischenergebnissen beschränken, da die Modellevaluation noch nicht abgeschlossen ist. Der Abschlussbericht wird voraussichtlich im Spätsommer 2001 vorliegen.
}

losophie in den Vordergrund der Arbeit: Im Rahmen von Case Management wird die Rolle des Klienten neu bestimmt. Er ist „Herr des Verfahrens“, gleichberechtigter Partner im gemeinsam strukturierten Hilfeprozess. Die Verfolgung z.B. von Zielen, die nicht wirklich diejenigen des Klienten sind, läuft unweigerlich ins Leere, eine von ihm miterstellte und -getragene Planung hat hingegen mehr Verbindlichkeit, fördert Compliance, Selbstverantwortung und Selbstwirksamkeit.

Eine weitere Neuerung liegt in der Fokussierung auf Planung: Während sich klassische Sozialarbeit z.T. in problem-reaktivem Handeln erschöpft, will Case Management die Richtung umkehren und zielgerichtet Hilfe planen sowie den Prozess (und damit auch die eigene Effektivität) laufend überprüfen.

\section{Klienten im Hilfeplanverfahren}

Von Mai 1997 bis Juni 1999 wurden von den Case Managern insgesamt 1420 Klienten betreut, die - im Vergleich zu Klienten z.B. der ambulanten Beratungsstellen - deutlich größere Probleme aufwiesen [3-5]. Die Klienten waren im Durchschnitt 39 Jahre alt und langjährig abhängig (65,5\% über 10 Jahre). $\mathrm{Zu}$ einem guten Drittel wurden Frauen betreut, wobei der relativ hohe Frauenanteil größtenteils auf den überproportionalen Anteil unter den Drogenabhängigen $(45,1 \%)$ zurückzuführen ist (in der Gruppe der Alkoholabhängigen waren $26,2 \%$ Frauen).

Die Problemlagen der Klienten waren vielfältig: Knapp vier Fünftel der Klienten waren arbeitslos, und das seit durchschnittlich 40 Monaten. Etwa ein Drittel verfügte dauerhaft über keine sichere Wohnsituation. Etwa 60\% der betreuten Klienten waren von Alkohol und 34,5\% von illegalen Substanzen abhängig. Wenngleich die meisten Klienten in der Vergangenheit bereits suchtbezogene Hilfen in Anspruch genommen hatten $(78,7 \%)$, so war es - angesichts der erfassten Konsumdauer und Problemlagen - doch überraschend, dass gut ein Fünftel bisher noch nie suchtspezifische Hilfen genutzt hatte.

Für gut vier Fünftel der Klienten $(81,7 \%)$ wurden typische suchtassoziierte somatische Erkrankungen, wie z.B. Hepatitis, (andere) Lebererkrankungen und Polyneuropathie, festgestellt. Zwei Drittel litten unter psychischen Störungen wie z.B. Persönlichkeitsstörungen, Depressionen und Angststörungen. Und bei fast allen Klienten lagen - zusätzlich zum Suchtproblem - gleichzeitig somatische und psychische Erkrankungen vor (85,7\%).

Hinsichtlich der Umsetzung von Hilfeplanung war zu untersuchen, ob diese schwer beeinträchtigten Klienten willens und in der Lage waren, an gemeinsamen Planungsprozessen mitzuwirken. Denn im Verlauf des Modells hatten die Case Manager wiederholt darauf hingewiesen, dass gerade für besonders schwer beeinträchtigte Klienten oder für Klienten in akuten Krisen kein Hilfeplan erstellt werden könne, insbesondere nicht als Ergebnis gemeinsamer Beratungen von Klient und Case Manager. Für einen anderen Teil der Klienten sei zudem keine Hilfeplanung notwendig, da bei kurzen Betreuungszeiten i.d.R. nur ein geringer Klärungsbedarf bestünde („zu aufwändig“, „nicht nötig“). 
Tatsächlich wurde nicht für alle, sondern nur für ein gutes Drittel der Klienten (543 Klienten, 38,2\%) eine Hilfeplanung durchgeführt. Mit zunehmender Erfahrung der Mitarbeiter mit der Methode stieg der Einsatz von Hilfeplanung an: Wurden im ersten Jahr für nur 35,7\% der Klienten Planungsverfahren eingeleitet, war das im zweiten Jahr schon für $42,7 \%$ der Fall. Um die o. g. Einschätzungen der Mitarbeiter zu bewerten, wurden die Daten der Klienten, für die ein Hilfeplanverfahren eingeleitet wurde, mit jenen ohne Hilfeplan verglichen.

Hierbei zeigte die Analyse soziodemographischer, sucht- und behandlungsbezogener Daten auf den ersten Blick keine nennenswerten Unterschiede zwischen Klienten mit und ohne Hilfeplanung. Um gleichwohl den beschriebenen Hinweisen der Mitarbeiter nachzugehen, wurden festgestellte Abweichungen $^{2}$ auf ihre statistische Bedeutsamkeit hin überprüft. Im Ergebnis ergaben sich einige, wenn auch meist nicht signifikante Unterschiede, die im Folgenden kurz beschrieben werden sollen.

Hilfeplanklienten waren zu Beginn der Betreuung etwas seltener in festen Partnerbeziehungen $(27,1 \%$ vs. $32,6 \%$ der Klienten, für die kein Hilfeplan angelegt wurde) und etwas öfter allein lebend (51,3\% vs. 44,5\%). Lebten sie nicht allein, so teilten sie sich die Wohnung etwas häufiger mit anderen Suchtkranken (58,3\% vs. $47,3 \%$ ) und hatten ebenfalls $\mathrm{zu}$ einem größeren Teil in den letzten zwei Jahren vorwiegend suchtbezogene Kontakte. Sie waren etwas seltener wohnungslos oder auf Notunterkünfte angewiesen.

Ihr Einkommen bestritten Hilfeplanklienten etwas seltener aus Lohn und Gehalt (4,3\% vs. 10,2\%) und aus Rente/Pension (5,7\% vs. $10,4 \%)$, dafür etwas häufiger aus Sozialhilfe $(35,2 \%$ vs. $27,7 \%$ ). Längerfristig betrachtet konnten sie auch weniger häufig auf ein regelmäßiges Einkommen zurückgreifen (53,9\% vs. $39,1 \%)$.

Eine Hilfeplanung wurde etwa gleich oft, je zu gut einem Drittel mit Alkohol- und mit Drogenabhängigen durchgeführt. Vergleicht man die Konsumdauer, zeigten sich keine nennenswerten Unterschiede: Hilfeplanklienten waren durchschnittlich ein knappes Jahr länger abhängig, darunter auch zu etwas größeren Anteilen bereits seit mehr als 15 Jahren ( $40,6 \%$ vs. $35,5 \%$ in der Gruppe ohne Hilfeplan). Bezüglich suchtspezifischer Behandlungserfahrungen zeigten sich in den beiden Klientengruppen keine wesentlichen Unterschiede, ebenso wie im Hinblick auf somatische Erkrankungen und psychische Störungen.

Insgesamt lieferte die klientenbezogene Datenanalyse also keine Hinweise für die Vorbehalte der Case Manager, dass klientenseitige Beeinträchtigungen bzw. krisenhafte Situationen einer Initiierung von Hilfeplanung entgegenstehen. Wie gezeigt, konnten (fast) keine relevanten Unterschiede

\footnotetext{
2 Prozentunterschiede wurden ab einer Differenz von $10 \%$ genauer untersucht, teilweise wurden auch geringere Prozentunterschiede auf Signifikanz geprüft. Dazu wurde bei $2 \times 2$ Tabellen als Assoziationsmaß Cramers V verwendet, das ab einem Wert $>20$ als ausreichend aussagekräftig betrachtet wurde, sofern die Irrtumswahrscheinlichkeit $\mathrm{p}<.01$ war.
}

zwischen den Klienten mit und ohne Hilfeplanverfahren beobachtet werden. Die wenigen Unterschiede wiesen, wenn überhaupt, eher auf eine stärkere Belastung der Hilfeplanklienten hin.

Dieses Ergebnis wird auch dadurch gestützt, dass Hilfeplanklienten länger (319 vs. 230 Tage Gesamtbetreuungsdauer) und intensiver als Nicht-Hilfeplanklienten (durchschnittlich 31,5 Stunden vs. 14,2 Stunden) betreut wurden. Möglicherweise antizipierten die Case Manager schon früh den erhöhten Hilfebedarf und strukturierten diese (schwierigeren, längeren, umfassenderen) Betreuungsprozesse mittels Hilfeplanung.

Ob ein Hilfeplan angelegt wurde, hing demnach nicht von spezifischen Klientenmerkmalen ab, sondern war vermutlich auf andere Aspekte zurückzuführen. In einem weiteren Analyseschritt wurden deshalb regionale bzw. einrichtungsbezogene Rahmenbedingungen hinsichtlich ihrer Auswirkungen auf die Umsetzung von Hilfeplanung untersucht. Im Ergebnis zeigten sich auch hier keine relevanten Unterschiede zwischen Case Managern aus Einrichtungen der Suchtkrankenoder Drogenhilfe, der Größe der Kommune, dem Versorgungsgrad der Region sowie neuen oder alten Bundesländern.

Unterschiede konnten jedoch in Abhängigkeit von der Gesamtzahl der von einem Case Manager betreuten Klienten beobachtet werden. Zunächst ist festzuhalten, dass die Zahl der betreuten Klienten stark variierte. Die Case Manager betreuten im Verlauf der beiden Dokumentationsjahre zwischen 19 und 113 Klienten, im Durchschnitt 44. Dabei setzten sie in unterschiedlichem Ausmaß Hilfeplanung um: Der Anteil der Klienten, mit denen Hilfeplanung durchgeführt wurde, streute von $0 \%$ bis zu $80 \%$.

Case Manager mit sehr vielen Klienten nutzten das Hilfeplanverfahren deutlich seltener als Modellmitarbeiter, die eine geringere Zahl von Klienten betreuten: Die Case Manager, die hinsichtlich der Klientenzahl die Rangplätze 1-10 belegen, betreuten im Dokumentationszeitraum durchschnittlich 70 Klienten und legten für nur 26\% dieser Klienten Hilfepläne an. Vergleicht man damit die Werte der zehn Case Manager mit den geringsten Klientenzahlen, so wurden hier durchschnittlich 22 Klienten betreut, für die in $47 \%$ der Fälle Hilfeplanverfahren eingeleitet wurden.

Allerdings entpuppte sich die Formel „weniger Klienten/intensivere Betreuung = mehr Hilfeplanung“ als zu vordergründig. So hatte z.B. ein Case Manager mit nur 20 Klienten keinen einzigen Hilfeplan angelegt, während der Modellmitarbeiter mit der höchsten Klientenzahl (113) immerhin für die Hälfte seiner Klienten Hilfeplanung durchführte. Weitere Belege ergaben sich aus Detail-Betrachtungen z.B. von Case Manager A und B: Sie arbeiteten in derselben Großstadt, konnten somit auf dasselbe Versorgungssystem zurückgreifen und betreuten die gleiche Anzahl vergleichbar problembelasteter (drogenabhängiger) Klienten. Gleichwohl hatten sie im Rahmen ihrer Betreuungen in sehr unterschiedlichem Umfang Hilfeplanverfahren eingeleitet: Case Manager A hatte in $29 \%$ seiner Fälle, Mitarbeiter B hingegen in $70 \%$ der Fälle Hilfepläne angelegt. 
Die Ergebnisse legen zusammenfassend betrachtet nahe, dass zwar ein Zusammenhang zwischen der Anzahl betreuter Klienten und dem Einsatz des aufwändigen Hilfeplanverfahrens besteht, gleichwohl die Haltung der Mitarbeiter den wichtigsten Einflussfaktor für die Umsetzung dieses Kernelements von Case Management darstellte.

Die Case Manager, die über größere Erfahrung im Einsatz von Hilfeplänen verfügten, schätzten das Verfahren als sinnvoll und effektiv ein. Sie betonten, dass damit die Realisierung von Klientenbeteiligung unterstützt, Assessments systematischer und lückenloser durchgeführt und eine regelmäßige und intensivere Reflexion angeregt wurden. Eine zielgerichtetere Fallführung erleichtere die Arbeit, mache Erfolge für Mitarbeiter und Klienten deutlicher wahrnehmbar und Leistungen nach außen transparenter. Um diese positiven Effekte sicherzustellen und um Fähigkeiten und Akzeptanz für dieses Verfahren zu erhöhen, sollte für künftige Case-ManagementProgramme Hilfeplanung intensiv geschult werden.

\section{Beteiligte an der Hilfeplanung}

Die Mitwirkung der Klienten und die Abstimmung von Hilfen verschiedener, an der Versorgung beteiligter professioneller, aber auch privater Helfer stellen zentrale Elemente von Case Management dar. Beispielsweise beschreiben Raiff und Shore [6] „qualifizierte Pläne [als] nichts anderes als [den] Niederschlag ausgehandelter Übereinkünfte zwischen Case Managern, Klienten, Familienangehörigen und den übrigen Fachkräften“. Case Management für mehrfachbeeinträchtigte Klienten steht jedoch immer im Spannungsfeld von Selbstbestimmung der Klienten und ggf. fehlender Fähigkeit zur Beteiligung.

Auf Basis der für 543 Klienten vorliegenden Hilfepläne wurde geprüft, inwieweit Klienten aktiv an der Planung und Strukturierung der Hilfen beteiligt waren. Ist dies nicht gewährleistet, stellt Hilfeplanung kein abgestimmtes Verfahren dar, sondern hat eher den Charakter einer Arbeitsplanung für professionelle Helfer.

Im Ergebnis zeigten die analysierten Hilfepläne, dass die Klienten überwiegend an der Planung beteiligt waren: Etwas mehr als die Hälfte der Klienten war vollständig, ein weiteres Viertel mit Einschränkungen beteiligt. Diese ergaben sich z. B. aufgrund begrenzter kognitiver Fähigkeiten, akuter Konsumphasen, begrenzter Motivation o.a. Nur jeder fünfte Klient war nicht an der Hilfeplanung beteiligt. Da die Klientenbeteiligung zentrales Umsetzungsmerkmal von Case Manage-

Tab. 1 Anzahl weiterer Beteiligter an den Hilfeplanungen ( $n=543)$

\begin{tabular}{lrr}
\hline Anzahl der Beteiligten & Anzahl der Pläne & in \% \\
\hline keiner & 185 & 34,1 \\
einer & 137 & 25,2 \\
zwei & 95 & 17,5 \\
drei & 71 & 13,1 \\
vier & 38 & 7,0 \\
fünf & 9 & 1,7 \\
sechs & 8 & 1,5 \\
\hline
\end{tabular}

ment ist, sollte die Nicht-Beteiligung explizit begründet werden. Von den Mitarbeitern wurden als Gründe insbesondere gesundheitliche Einschränkungen wie z.B. „beginnendes Korsakow-Syndrom“ und konsumbedingte Eigenschaften von Klienten (wie „Sprunghaftigkeit“ oder „Zurzeit wieder stark unter Alkoholeinfluss, teilweise nicht ansprechbar“) genannt.

Außerdem wurde untersucht, ob und welche anderen professionellen Helfer an der Planung beteiligt waren. Dazu sollen in einem ersten Schritt die vermittelnden Instanzen für die Hilfeplanklienten betrachtet werden: Gut ein Fünftel war ohne Vermittlung (104 Klienten, 22,0\%) und 63 Klienten $(13,3 \%)$ durch Angehörige, Freunde oder Bekannte zu den Case Managern gelangt. Der wichtigste zuweisende Versorgungssektor war der medizinische Bereich, aus dem fast ein Viertel der Klientel kam (Gesundheitsamt/sozialpsychiatrischer Dienst: 9,3\% Allgemeinkrankenhaus: 8,9\%, ärztliche Praxis: 6,6\%). Aus der Suchtkrankenhilfe wurden insgesamt 22,0\% der Klienten (Fachklinik und Suchtberatung: je 6,4\%, Substitutionsambulanz und niedrigschwellige Angebote: je 4,2\%, Betreutes Wohnen/Nachsorge: 0,8\%) vermittelt.

Betrachtet man nun, wie viele Personen bzw. Institutionen an Hilfeplanungen teilgenommen hatten, so zeigt sich, dass nur für zwei Drittel der Klienten weitere Beteiligte dokumentiert wurden (vgl. Tab. 1). Im Durchschnitt waren neben den Case Managern 1,5 weitere Personen bzw. Institutionen am Hilfeplanverfahren beteiligt. In etwa einem Drittel der Fälle (185 Klienten, 34,1\%) hat außer den Case Managern niemand an den Hilfeplanungen mitgewirkt.

Insgesamt wurden 785 Personen bzw. Institutionen erfasst, die an der Betreuung von 358 Klienten beteiligt waren. Entsprechend der Zugangswege und der Problemlagen der Klienten handelte es sich dabei ganz überwiegend um ärztliche Praxen (bei 34,6\% der Klienten) und Krankenhäuser $(21,8 \%)$. Eine Beteiligung von Angehörigen oder anderen Bezugspersonen des privaten Umfelds ist nur dann realisierbar, wenn zu diesen Kontakte bestehen. Da dies nur für einen Teil der Klienten zutraf und 163 Klienten explizit keine Teilnahme ihrer Angehörigen (35,5\%) wünschten, ist hervorzuheben, dass immerhin in gut jedem vierten Fall Angehörige der Klienten an der Hilfeplanung beteiligt waren (134 Fälle, $29,2 \%)$.

Als weitere Beteiligte folgten das Sozialamt (20,4\%), Substitutionsambulanzen (13,7\%), psychiatrische Krankenhäuser $(11,7 \%)$, das Jugendamt $(9,5 \%)$. Andere Institutionen bzw. Personen waren nur selten beteiligt, darunter z. B. gesetzliche Betreuer (4,6\%).

In der Fachdiskussion spielen Hilfekonferenzen für die Steuerung komplexer sozialer Dienstleistungen eine herausragende Rolle. Eine Modellannahme zielte deshalb darauf, dass auch Hilfen für mehrfachbeeinträchtigt Abhängige effektiver erbracht werden können, wenn Bezugspersonen des privaten Umfelds und professionelle Helfer ihre Vorgehensweise mittels Hilfekonferenzen abstimmen, Ziele und Maßnahmen vereinbaren. In diesem Sinne sollten Hilfekonferenzen immer dann durchgeführt werden, wenn außer dem Case Manager und dem Klienten noch zwei weitere (professionelle oder private) Helfer an der Gesamtbetreuung der Klienten beteiligt waren. Dies war bei ca. vier Fünftel der Klienten der Fall. 
Hierbei zeigten vertiefte Analysen, dass Hilfekonferenzen ausschließlich im Kontext von Hilfeplanungsprozessen stattfanden, im Rahmen der ersten Hilfepläne in 55 Fällen (10,1\%). An diesen Betreuungen waren außer Case Managern und Klienten weitere 106 Personen bzw. Hilfeinstanzen beteiligt. Obwohl Angehörige in gut einem Viertel der Fälle als Planungsbeteiligte genannt wurden, nahmen sie nur an fünf Hilfekonferenzen teil. Am häufigsten engagierten sich (Jugend-)Ämter (20-mal), gefolgt von medizinischen Leistungsanbietern (18-mal) und Einrichtungen der Suchtkrankenhilfe (13-mal). Gesetzliche Betreuer nahmen nur bei neun Klienten an Hilfekonferenzen teil (obgleich für 45 Hilfeplanklienten eine gesetzliche Betreuung bestand). Im Durchschnitt haben vier Personen an den Hilfekonferenzen teilgenommen.

Im bisherigen Verlauf des Modellprogramms hat sich die Vorgabe an die Case Manager, bei möglichst vielen Klienten Hilfekonferenzen durchzuführen, als schwierig erwiesen. Die Gründe für diesen Befund sind vielfältig: Auf der einen Seite berichteten die Case Manager, dass andere Hilfeinstanzen u.a. aufgrund des zeitlichen und organisatorischen Aufwands oftmals nicht bereit waren, an Hilfekonferenzen teilzunehmen. So beteiligten sich z.B. niedergelassene Ärzte und Rechtsanwälte nur sehr selten an diesen Konferenzen, da sie für diese Leistung keine Gebühren geltend machen können.

Andererseits wies eine Vielzahl von Case Managern darauf hin, dass ihnen ein „Mandat“ fehle, um fallbezogen verbindliche Absprachen mit anderen Leistungsanbietern zu treffen und deren Einhaltung zu kontrollieren. Solange sie auf den guten Willen von Mitarbeitern anderer Einrichtungen und Dienste angewiesen seien, wären Hilfekonferenzen als Standard nicht umsetzbar, eine Zusammenarbeit würde häufig verweigert. Aus den genannten Gründen hat ein Teil der Case Manager erst gar nicht versucht, Hilfekonferenzen zu initiieren, sondern traf im Rahmen bilateraler Gespräche (häufig telefonisch) Absprachen mit anderen Leistungsanbietern.

Im bisherigen Verlauf des Modellprogramms stieß die Durchführung von Hilfekonferenzen jedoch nicht nur aufgrund der unzureichenden Kompetenzausstattung der Case Manager und der fehlenden Bereitschaft anderer Hilfeinstanzen, daran mitzuwirken, auf Schwierigkeiten. Hemmend wirkten sich zudem die unzureichende Erfahrung mit diesem Instrument, Scheu vor Transparenz, mangelnde Routine im Moderieren und Steuern von Gruppen sowie Abgrenzungstraditionen zwischen Einrichtungen und Trägern, Skepsis und Beharrungstendenzen etc. aus.

Falls Hilfekonferenzen durchgeführt wurden, waren damit positive Erfahrungen verbunden. Stellvertretend für diese Einschätzung soll aus dem Jahresbericht eines Case Managers zitiert werden: „Als besonders hilfreich muss auch der besondere Schwerpunkt der Kooperation und Koordination von Hilfen im Rahmen des Case Managements gesehen werden. So hat es sich besonders bewährt, die notwendigen Hilfen in Fallkonferenzen mit allen zuständigen Helfern (Beratern, Betreuern, Ärzten, Therapeuten) abzustimmen und ein gemeinsames weiteres Vorgehen zu verabreden. Diese verabredeten Hilfen in einem Hilfeplan zu dokumentieren, konsequent weiterhin nachzugehen und über einen zeitlichen Abstand immer wieder zu überprüfen, ist sehr effektiv“ [7].
Tab. 2 Ziele mit erster Priorität

\begin{tabular}{|c|c|c|}
\hline Ziele/Zielbereich & $\begin{array}{l}\text { 1. Hilfe } \\
(n=28 \\
\text { Anzahl }\end{array}$ & $\begin{array}{l}\text { läne } \\
\text { in \% }\end{array}$ \\
\hline $\begin{array}{l}\text { Sicherung oder Verschaffung einer } \\
\text { Wohnung, Unterkunft o. ä. }\end{array}$ & 45 & 16,0 \\
\hline Entgiftung & 43 & 15,3 \\
\hline Therapie & 34 & 12,1 \\
\hline $\begin{array}{l}\text { Vermittlung/Organisation/Unterstützung } \\
\text { bzgl. medizinischer Hilfe }\end{array}$ & 31 & 11,0 \\
\hline Konsumreduzierung & 29 & 10,3 \\
\hline Einkommen/materielle Situation & 19 & 6,8 \\
\hline Koordination verschiedener Maßnahmen & 17 & 6,0 \\
\hline Kontakthalten mit dem Klienten & 13 & 4,6 \\
\hline $\begin{array}{l}\text { Organisation bzw. Unterstützung bzgl. } \\
\text { Substitution }\end{array}$ & 9 & 3,2 \\
\hline Beratung zu Beziehungsproblemen & 8 & 2,8 \\
\hline Hilfen bzgl. Arbeit(ssuche) & 6 & 2,1 \\
\hline Unterstützung bei Problemen mit der Justiz & 4 & 1,4 \\
\hline $\begin{array}{l}\text { Vermittlung in Nachsorge/Stabilisierung von } \\
\text { Abstinenzphase }\end{array}$ & 2 & 0,7 \\
\hline sonstiges & 21 & 7,5 \\
\hline Gesamt & 281 & 100,0 \\
\hline
\end{tabular}

\section{Ziele, Maßnahmen und Zuständigkeiten}

\section{Zielplanung und Prioritätensetzung}

$\mathrm{Zu}$ einer strukturierten Hilfeplanung gehören vier wesentliche Schritte: Zielplanung, Prioritätenzuordnung, Definition von Maßnahmen bzw. Verfahren, Festlegung von zuständigkeiten und Überprüfungszeiten [8]. Das Modellprogramm sah deshalb vor, dass die Case Manager an den Bedürfnissen der Klienten ausgerichtete und im Hinblick auf ihre Realisierung angemessene Ziele formulieren sollten. Angesichts der Zielgruppe stellte Abstinenz dabei häufig ein entfernteres Ziel dar. Andere (Teil-)Ziele konnten diesem vorgelagert und der aktuellen Situation der Klienten angemessener sein. Handlungsleitend sollte dabei die auf Schwoon [9] zurückgehende und später von Wienberg [10] modifizierte „Hierarchie der Interventionsziele“ sein.

Die Auswertung der Zielplanung ergab folgende Befunde: Eine Rahmenplanung lag in fast allen Fällen vor (95,2\%) und eine Rangreihe der Ziele wurde in $86,8 \%$ aller Hilfepläne aufgestellt. Davon unterschieden wurde bei fast $90 \%$ eine detaillierte Planung.

Analysiert man die in der Hilfeplanung mit erster Priorität formulierten Ziele (vgl. Tab. 2) zeigte sich, dass bei etwa einem Sechstel der Hilfeplanklienten zu Beginn der Betreuung das Problemfeld Wohnen im Vordergrund stand. Für 15,3\% der Klienten war zunächst eine Entgiftungsbehandlung zu vermitteln. Das lag vor allem darin begründet, dass ein stationärer Aufenthalt den Klienten eine Konsumpause ermöglichte, in der Veränderungsmotivation sich entwickeln und gefördert werden konnte. Erstaunlicherweise wurde bei etwa jedem achten Klienten auf eine stationäre Therapie hingearbeitet. Hierbei handelte es sich insbesondere um Klienten, die im Anschluss an eine Entgiftung oder an einen anderen Krankenhausaufenthalt in die Betreuung übernommen wurden. Nur bei etwa jedem neunten Klienten standen 
Behandlungen somatischer Erkrankungen oder psychischer Störungen an erster Stelle.

Konsumreduzierung oder -kontrolle stand bei etwa jedem zehnten Klienten im Vordergrund der Betreuung. Dieser Anteil erstaunt angesichts der vielen existentiellen Probleme der Klienten, denn nicht nur Konsumreduzierung, sondern auch klassische Ziele von Suchtberatung waren hier prioritär für die erste Hilfeplanung. Qualitative Auswertungen der Hilfepläne zeigten, dass es sich bei den Zielen im Bereich Konsum, Entgiftung, Substitution (und Gesundheit) größtenteils um Ziele auf einer der unteren Hierarchieebenen, z.B. zur Vermeidung von lebensgefährlichen Überdosierungen, handelt. Bei etwa zwei Fünfteln unterstützten die Case Manager ihre Klienten demnach bei der Sicherung des Überlebens, verhinderten oder verminderten schwere körperliche Folgeschäden oder sicherten die (soziale) Umgebung der Klienten gegen Beeinträchtigungen ab.

Außerdem ist zu berücksichtigen, dass zum einen Hilfeplanung i.d.R. nicht gleich zu Betreuungsbeginn einsetzte, so dass z.B. die Sicherung der Wohnung oder die Vermittlung eines Schlafplatzes oder die sofortige Vermittlung in ein Krankenhaus wegen einer akuten Behandlungsbedürftigkeit zu diesem Zeitpunkt häufig schon erfolgt waren. Zum anderen konnten die an späterer Stelle genannten Ziele - trotz der Prioritätensetzung - ähnlich dringlich gewesen sein.

Die für fast ein Drittel der Klienten genannten Betreuungsziele in den Bereichen Wohnen, Einkommen, Arbeit, justitielle Situation, aber auch die Ziele im Bereich „Beziehung“ waren vor allem auf der nächsthöheren Ebene angesiedelt, d.h. betrafen die Verhinderung bzw. Verminderung sozialer Desintegration.

Ziele hinsichtlich Therapie und Nachsorge gehörten zu einer noch höheren Hierarchieebene. Diese Klienten wiesen z.T. längere Abstinenzphasen auf, zeigten Einsicht in die Grunderkrankung und akzeptierten den eigenen Behandlungs- bzw. Hilfebedarf. Angesichts der Zielgruppe des Modellprogramms überrascht es nicht, dass nur für eine kleine Gruppe (6,7\%) derartige Ziele prioritär verfolgt wurden.

Für 10,6\% der Klienten standen definierte Aufgaben von modellgefördertem Case Management, z. B. „Koordination von Hilfen“ und - vorerst „Kontakthalten“ - im Vordergrund.

Die Case Manager beschrieben Schwierigkeiten hinsichtlich der Zieldefinition. Unklar war z.B., welcher Konkretisierungsgrad angemessen ist, welcher Zeithorizont avisiert werden sollte und wie Ziele von Maßnahmen abzugrenzen waren.

\section{Maßnahmen und Zuständigkeiten}

Die Definition von konkreten Arbeitsschritten lehnte sich eng an die Ziele an und bezog sich im Wesentlichen auf deren konkrete Erfüllung: Genannt wurden z.B. „Arzt anrufen; Klient zu Arzt begleiten; Klient auf Entgiftungsstation anmelden; Termin bei Schuldnerberatung vermitteln; Konsum reduzieren und nur noch abends oder nicht allein trinken; in Krisen den Case Manager anrufen etc.“.
Für die Planung war neben dem „was“ auch wichtig, wer für die ausgewählte(n) Maßnahme(n) zuständig ist. Für drei von fünf definierten Maßnahmen wurden Zuständigkeiten bestimmt, wobei insgesamt 795-mal Personen bzw. Institutionen genannt wurden (das entspricht durchschnittlich etwa 2,4 Nennungen pro Hilfeplan).

Bei der Festlegung der Zuständigkeiten wurden an erster Stelle die Case Manager genannt (in etwa $4 / 5$ der Pläne), was bestätigt, dass sie sich meist nicht auf Vermittlungen und koordinative Tätigkeiten beschränken, sondern auch selbst Leistungen übernehmen mussten. Die Klienten waren nur in zwei Drittel der Hilfepläne selbst für bestimmte Aufgaben zuständig, was angesichts der angestrebten Klientenbeteiligung als problematisch zu werten ist.

Die verschiedenen professionellen Hilfeinstanzen übernahmen fast ebenso oft Aufgaben wie die Case Manager. In Abstimmungsprozessen kann es demnach gelingen, Aufgaben zu delegieren und einen „Verbund von Helfern“ zu schaffen. Die Ergebnisse verdeutlichen, dass der hohe konzeptionelle Anspruch von gemeinsamer Hilfeplanung aller an der Versorgung eines Klienten Beteiligten zwar auf schwierige Umsetzungsbedingungen traf, es den Case Managern im Modellprogramm gleichwohl gelang, erste Erfolge zu erzielen.

Angehörige, die an einem Viertel der Planungsprozesse teilgenommen hatten, übernahmen in nur sehr eingeschränktem Ausmaß entsprechende Aufgaben. Die Case Manager wiesen erläuternd darauf hin, dass Angehörige häufig zunächst entlastet werden wollten und es begrüßten, wenn andere Hilfeinstanzen aktiv wurden. Zum Teil bemühten sich die Case Manager hier um die Stabilisierung familiärer Beziehungen, indem sie Angehörige unterstützten und ihnen ermöglichten, sich einmal zurückzunehmen.

\section{Überprüfung und Unterzeichnung}

Der Case Management-Prozess wird im Rahmen des Kooperationsmodells nachgehende Sozialarbeit durch regelmäßige (Re-)Assessments und Fortschreibungen der Hilfeplanung strukturiert. Dabei besteht die Vorgabe, etwa alle drei Monate die Situation des Klienten, seine Entwicklung und die Wirkung der Maßnahmen zu überprüfen, um dann ggf. die Zielund Hilfeplanung zu modifizieren.

Um ein derartiges Monitoring von vornherein in die Hilfeplanung zu integrieren, sollte am Ende der Planung ein Überprüfungstermin vereinbart werden. Tatsächlich dokumentierten die Case Manager in drei Viertel der Fälle entsprechende Daten, die im Durchschnitt knapp drei Monate nach dem Planungstermin lagen ( $\varnothing 86$ Tage).

Schließlich sollten sämtliche Vereinbarungen der Hilfeplanung mit der Unterschrift aller Beteiligten bestätigt und die Verbindlichkeit der Absprachen durch die schriftliche Form gesteigert werden. Hier zeigte sich jedoch erneut die unzureichende Routine der Case Manager und die fehlende Vertrautheit anderer Versorgungsbeteiligter mit einem derartigen Verfahren: Nur 8,7\% der Pläne waren überhaupt von den Klienten und nur $9(1,7 \%)$ von weiteren Beteiligten unterschrieben(vgl. Tab.3). Das Vorgehen wurde zwar meist mit den Klienten erörtert, jedoch seltener tatsächlich gemeinsam 
geplant und nur in Ausnahmefällen verbindlich mit ihnen vereinbart. Auch die Absprachen mit Mitarbeitern anderer Hilfeinstanzen waren öfter informeller Natur. Selbst die 55 durchgeführten Hilfekonferenzen wurden i.d. R. nicht mittels des Hilfeplans dokumentiert oder in anderer Form schriftlich und für alle Beteiligten einsehbar protokolliert.

Dazu berichteten die Case Manager, dass sie sich oft nicht getrauten, Kollegen formell einzubinden; auch bestanden durchaus auf allen Seiten - Ängste vor Transparenz, Verbindlichkeit und (möglicher) Kontrolle. Es wurde deutlich, dass die Steuerung und Abstimmung von Hilfeprozessen der Übung und Gewöhnung bedarf. Dabei geht es um systematisches Strukturieren und nachvollziehbares Dokumentieren, um das Praktizieren moderativer und fallführender Funktionen sowie die Herausbildung von Modi der ZusammenArbeit. Es sollten jedoch nicht nur einzelne Helfer neue Wege der Kooperation umsetzen („bottom-up“), wichtig wäre darüber hinaus eine strukturelle Entsprechung auf Trägerebene, die Zusammenarbeit fordert und fördert („top-down“).

\section{Diskussion}

Hilfeplanung ist nicht nur ein Element unter vielen, sondern das Prinzip aller Arbeitsschritte von Case Management. Sie strukturiert den gesamten Betreuungsprozess und ist die Grundlage für die Steuerung von Abstimmungsprozessen, das Monitoring, also die laufende Fallkontrolle, die Überprüfung von Zielen und (abschließende) Bewertung von Betreuungsverlauf und Zielerreichung. Gleichzeitig zielen Case Management und Hilfeplanung auf eine Veränderung von Sozialarbeit (hier in der Suchtkrankenhilfe), indem bisher ungewohnte Arbeitsprinzipien, wie z.B. Planung und Abstimmung von Hilfen, eine so nicht flächendeckend praktizierte Form der Klientenbeteiligung und schließlich eine transparente Beschreibung des Betreuungsprozesses eingefordert werden. An dieser Stelle zeigten sich im Modellprogramm erwartungsgemäß große Umsetzungsprobleme: Insgesamt wurde nur für etwa ein Drittel aller Klienten ein Hilfeplanverfahren eingeleitet, entsprechende Fortschreibungen waren noch seltener. Die Case Manager machten vor allem klientenseitige Gründe für das zögerliche Umsetzen des Hilfeplanverfahrens verantwortlich: Hervorgehoben wurden insbesondere $\mathrm{zu}$ große Beeinträchtigungen, geringe Verlässlichkeit, mangelnde Stabilität, kognitive Begrenzungen der Klienten etc. Tatsächlich wurden eher für vergleichsweise schwerer belastete Klienten, die auch insgesamt intensiver betreut wurden, häufiger Hilfeplanverfahren initiiert.
Der Einsatz von Hilfeplanung hing des Weiteren mit der Anzahl der betreuten Klienten zusammen, wenngleich eine geringere Klientenzahl keine hinreichende Bedingung für mehr Hilfeplanung war. Vielmehr hatten Einstellung und Bereitschaft der Mitarbeiter zu dem Verfahren entscheidenden Einfluss auf den Einsatz von Hilfeplanung.

Die Inhaltsanalysen von Hilfeplänen wiesen eine Vielfalt von Zielstellungen auf, die sich häufig auf die Sicherung materieller Grundlagen oder gesundheitlicher Probleme sowie Konsumveränderungen bezogen. Ein wichtiger Aspekt in vielen Planungen stellte die Koordination und Abstimmung von Hilfen dar. Deutlich wurde, dass die Qualität der (Arbeits-)Beziehung zwischen Klient und Mitarbeiter ein Dreh- und Angelpunkt der Arbeit ist, ohne den die Klienten nicht zu Veränderungen zu motivieren sind. Die Analysen verdeutlichten aber auch Schwierigkeiten bei der Identifikation, Abstimmung und Formulierung von Zielen. Die Planungen legten Aufgaben und Zuständigkeiten fest, wobei es den Case Managern gelungen war, in hohem Maß andere Hilfeinstanzen einzubeziehen und Aufgaben zu delegieren. Leider konnten Angehörige bisher nur wenig für eine Übernahme von Aufgaben motiviert werden; hier mussten die Case Manager z. T. eher zu einer (Arbeits-)Entlastung beitragen.

Eine Hilfeplanung sollte damit abgeschlossen werden, dass alle Beteiligten einen Termin zur Überprüfung von Planung und Entwicklung vereinbaren, den Hilfeplan unterschreiben und eine Kopie davon als „Protokoll“ der Vereinbarung erhalten. Dieses Ideal wurde nicht erreicht. Als problematisch erwiesen sich dabei weniger die Klienten als die professionellen Helfer, den Case Manager eingeschlossen, die von sich aus auf Unterschriften verzichteten und die Hilfepläne nicht für die Klienten oder für andere Beteiligte vervielfältigten.

Hilfeplanung soll nicht nur den Arbeitsprozess von Case Managern und Klienten, sondern auch die Abstimmung von professionellen Hilfen strukturieren. Hilfekonferenzen, in denen alle an einem Fall Beteiligten (inkl. des Klienten) das Vorgehen gemeinsam beraten und Zuständigkeiten abstimmen, wurden als ein geeignetes Mittel zu diesem Zweck angesehen. Doch auch diese Annahme wurde durch die bisherigen Erkenntnisse nicht bestätigt. Die Case Manager wiesen auf den hohen zeitlichen und organisatorischen Aufwand sowie die Vorteile (telefonischer) bilateraler Absprachen hin. Zudem fehlte ihnen schon für die Einberufung einer Hilfekonferenz und erst recht für die Verbindlichkeit der Absprachen und deren Kontrolle die (formale) Kompetenz. Als ebenfalls hemmend wirkten sich die unzureichende

\begin{tabular}{lcc}
\hline Unterzeichner & Anzahl & in \% \\
\hline Case Manager und Klient & 39 & 7,2 \\
Case Manager, Klient und professionelle Dritte & 6 & 1,1 \\
Case Manager, Klient, private und & 1 & 0,2 \\
professionelle Dritte & 2 & 0,4 \\
Case Manager und professionelle Dritte & 1 & 0,2 \\
Nur Klient & 104 & 19,2 \\
Nur Case Manager & 390 & 71,8 \\
Ohne Unterschrift & 543 & 100,0 \\
Gesamt & & \\
\hline
\end{tabular}

Tab. 3 Unterzeichner von Hilfeplänen $(n=543)$ 
Erfahrung mit diesem Instrument, Scheu vor Transparenz, mangelnde Routine in Moderations- und Steuerungsaufgaben, Abgrenzungstraditionen zwischen Einrichtungen und Trägern, Skepsis und Beharrungstendenzen etc. aus. Gelang es Hilfekonferenzen durchzuführen, wurden durchweg positive Erfahrungen gesammelt und die Annahme bestätigt, dass gemeinsame durch Hilfekonferenzen gestützte Hilfeplanung mehr Verfahrensklarheit bringt und am Ende auch Zeit spart.

Grundsätzlich bestätigten die Case Manager, die eine größere Anzahl von Hilfeplanungen durchgeführt hatten, dass das Verfahren sinnvoll und prozessunterstützend ist. Es fördert die Realisierung von Klientenbeteiligung, gestaltet Assessments systematischer und lückenloser und ermöglicht eine regelmäßige und intensivere Reflexion. Durch den Einsatz von Hilfeplanung wurde die zielgerichtete Fallführung erleichtert, Erfolge für Mitarbeiter und Klienten wurden deutlicher wahrnehmbar und Leistungen für Außenstehende transparenter.

Insgesamt konnten im Modellprogramm wichtige Erfahrungen mit Case Management und Hilfeplanung gesammelt werden, die im Wesentlichen die Bedeutung und Sinnhaftigkeit des Verfahrens bestätigten. Gleichwohl sind Schwierigkeiten in der Umsetzung aufgetreten, deren Lösung sowohl ein Umdenken auf Seiten der Case Manager als auch auch Einstellungsänderungen seitens der Kooperationspartner erfordern.

\section{Literatur}

${ }^{1}$ Bundesministerium für Gesundheit. Vorschlag für ein Bund/ Länder-Konzept zur Verbesserung der Drogen- und Suchthilfe insbesondere für „chronisch abhängige“ Suchtkranke. Unveröffentlichtes Manuskript. Bonn, 1995

2 Wendt WR. Case Management im Sozial- und Gesundheitswesen. Eine Einführung. Freiburg, 1997

${ }^{3}$ Oliva H, Görgen W, Schlanstedt G, Schu M, Sommer L. Kooperationsmodell nachgehende Sozialarbeit. Zwischenbericht im Auftrag des Bundesministeriums für Gesundheit. Köln, 1998

${ }^{4}$ Oliva H, Görgen W, Schlanstedt G, Schu M, Sommer L. Kooperationsmodell nachgehende Sozialarbeit. 2. Zwischenbericht im Auftrag des Bundesministeriums für Gesundheit. Köln, 1999

${ }^{5}$ Oliva H, Görgen W, Schlanstedt G, Schu M, Sommer L. Kooperationsmodell nachgehende Sozialarbeit. 3. Zwischenbericht im Auftrag des Bundesministeriums für Gesundheit. Köln, 2000

${ }^{6}$ Raiff NR, Shore BK. Fortschritte im Case Management. Freiburg, 1997

${ }^{7}$ AWO Kreisverband Bremerhaven e.V. Jahresbericht 1998 des Suchtberatungszentrums. Bremerhaven, 1999

${ }^{8}$ Ballew JR, Mink G. Was ist Case Management? In: Wendt WR (Hrsg). Unterstützung fallweise - Case Management in der Sozialarbeit. Freiburg, 1995

${ }^{9}$ Schwoon DR. Motivation - ein kritischer Begriff in der Behandlung Suchtkranker. In: Wienberg, G (Hrsg). Die vergessene Mehrheit: Zur Realität der Versorgung alkohol- und medikamentenabhängiger Menschen. Bonn, 1992

${ }^{10}$ Wienberg G. Die vergessene Mehrheit - Struktur und Dynamik der Versorgung Abhängigkeitskranker in der Bundesrepublik. In: Aktion psychisch Kranke (Hrsg). Gemeindepsychiatrische Suchtkrankenversorgung - regionale Vernetzung medizinischer und psychosozialer Versorgungsstrukturen. Köln, 1997; Tagungsberichte Band 21

\section{Martina Schu}

FOGS GmbH

Prälat-Otto-Müller-Platz 2

50670 Köln

E-mail: schu@fogs-gmbh.de 